\title{
Edaravone Suppresses Reperfusion Injury following Leg Ischemia in Rats: A Transmission Electron Microscopic Study
}

\author{
Mitsuhiro Yamamura, MD, FICA ${ }^{1}$ Yuji Miyamoto, MD $^{1}$ \\ Masaaki Ryomoto, $\mathrm{MD}^{1}$ Shinya Fukui, MD ${ }^{1}$ \\ ${ }^{1}$ Department of Cardiovascular Surgery, Hyogo College of Medicine, \\ Hyogo, Japan \\ Int J Angiol 2013;22:267-270.
}

\author{
Masataka Mitsuno, $\mathrm{MD}^{1}$ Hiroe Tanaka, MD ${ }^{1}$
}

\author{
Address for correspondence Mitsuhiro Yamamura, MD, FICA, \\ Department of Cardiovascular Surgery, Hyogo College of Medicine, 1-1 \\ Mukogawa-cho, Nishinomiya City, Hyogo 663-8501, Japan \\ (e-mail: yamachan@hcm-mail.hyo-med.ac.jp).
}

\begin{abstract}
It is well known that free radicals cause reperfusion injury following leg ischemia. We showed that the free radical scavenger, edaravone (Radicut, Mitsubishi Tanabe Pharma Co., Osaka, Japan), might suppress reperfusion injury in rat. In this study, we used transmission electron microscope (TEM) to investigate how edaravone suppresses reperfusion injury by focusing on glycogen granules in the lower extremity muscles. Male Lewis rats $(582 \pm 35 \mathrm{~g}$ ) were intraperitoneally injected with edaravone $(3.0 \mathrm{mg} /$ $\mathrm{kg}$, edaravone group, $n=5$ ) or the same dose of saline (control group, $n=5$ ). The rat reperfusion injury models were induced by clamping the bilateral common femoral arteries for 5 hours and then declamping. The muscles were harvested at 5 hours after the start of reperfusion. Under a TEM (JEM-1220, Nippon Denshi Co., Tokyo, Japan), we counted the number of glycogen granules at $\times 50,000$ magnification on each five different fields. The TEM sections from the control group showed a marked loss of glycogen granules and swollen mitochondria. In contrast, the TEM sections from the edaravone group showed numerous glycogen granules and normal mitochondria. The mean density of glycogen granules in the edaravone group was significantly higher than that in the control group $\left(88.5 \pm 5.3\right.$ vs. $16.4 \pm 3.1$ particles $\left./ \mu m^{2}, p<0.001\right)$. Our TEM results confirmed that edaravone suppresses reperfusion injury following leg ischemia by maintaining the glycogen granules in muscles.
\end{abstract}

In 1960, Haimovici reported two postoperative cases of myonephropathic metabolic syndrome (MNMS). ${ }^{1}$ Since this report, it has been clear that free radicals cause lower extremity reperfusion injury and MNMS. ${ }^{2}$ However, there was no commercially available free radical scavenger. Edaravone (3-methyl-1-phenyl-2-pyrazoline-5-one, $\mathrm{C}_{10} \mathrm{H}_{10} \mathrm{~N}_{20}$, molecular weight $=174.2$, Radicut, Mitsubishi Tanabe Pharma Co., Japan) is the first free radical scavenger, and it is effective for the treatment of patients with acute cerebral infarction. ${ }^{3}$ We have shown that edaravone might suppress reperfusion injury in rat models., ${ }^{4,5}$ And our recent study of periodic acid Schiff (PAS) staining indicated that edaravone maintained high glycogen storage in lower extremity muscles. ${ }^{6}$

In this study, we used transmission electron microscope (TEM) focusing on glycogen granules in the lower extremity muscles, to further understand the mechanisms how edaravone suppresses reperfusion injury.

\section{Materials and Methods}

\section{Rat Reperfusion Injury Model}

The rat reperfusion injury model was made under a microscope (OME-J\&N J73507R, Olympus, Tokyo, Japan), as
Copyright @ 2013 by Thieme Medical Publishers, Inc., 333 Seventh Avenue, New York, NY 10001, USA. Tel: +1(212) 584-4662.
DOI http://dx.doi.org/ 10.1055/s-0033-1357261. ISSN 1061-1711. 


\section{Control}

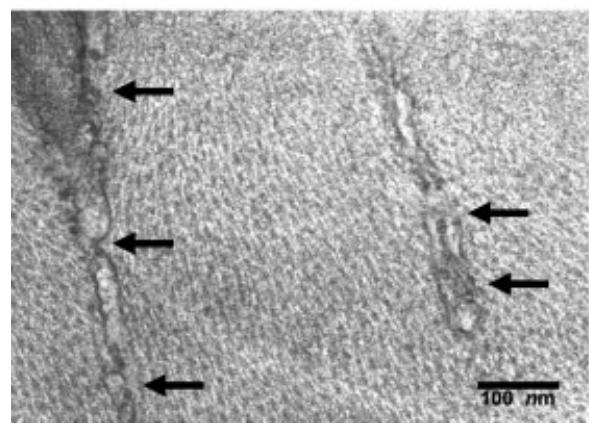

Edaravone

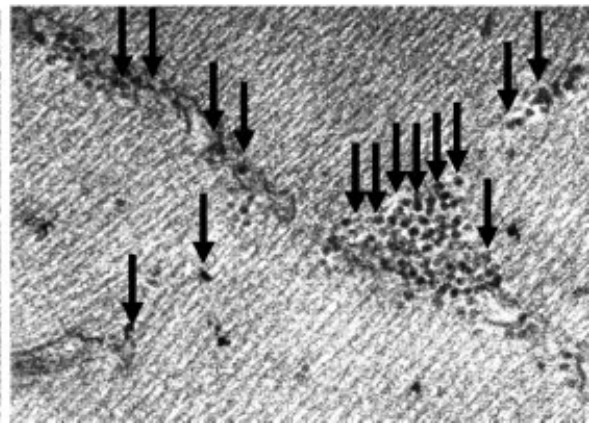

Fig. 1 Transmission electron microscopic image of glycogen granules, control group versus edaravone group. Original magnification $\times 50,000$. Each arrow indicates glycogen granules.

previously described. ${ }^{4-6}$ Male Lewis rats $(582 \pm 35 \mathrm{~g})$ were divided into two groups. Before the operation, they were intraperitoneally injected with edaravone $(3.0 \mathrm{mg} / \mathrm{kg}$, edaravone group, $n=5$ ) or the same dose of saline (control group, $n=5$ ). Complete general anesthesia was induced by intraperitoneal administration of $30 \mathrm{mg} / \mathrm{kg}$ of sodium pentobarbital (Nembtal, Abbot Co., IL). To block these collateral supplies completely, both deep femoral arteries and epigastric arteries were ligated. Then, the bilateral common femoral arteries were clamped for 5 hours and then declamped.

All animal procedures complied with the criteria of Hyogo College of Medicine Animal Research Committee criteria (No. B-10-003, B-11-202).

\section{Transmission Electron Microscopic Study}

At 5 hours after the start of reperfusion, the lower extremity muscles were immediately harvested and cut transversely with the diamond knife. Glycogen storage was defined as the number of glycogen granules on TEM (JEM-1220, Nippon Denshi Co., Tokyo, Japan). TEM photos at $\times 50,000$ magnification on each five different fields were taken by blinded researchers (R.F. and H.K.). We manually counted the glycogen granules on each photos. The density of glycogen granules (particles $/ \mu \mathrm{m}^{2}$ ) was measured and compared between the two groups.

\section{Statistical Analysis}

All results are expressed as mean \pm standard error of mean (SEM). The data were statistically analyzed using unpaired $t$ tests (Stat View J-4.5 for Macintosh, Abacus Concept, Berkeley, CA). A $p$ value of less than 0.05 was considered to indicate statistical significance.

\section{Results}

There were no operative deaths in either of the group. Representative TEM sections after reperfusion injury are shown in - Fig. 1. The TEM sections from the control group showed marked loss of glycogen granules and swollen mitochondria. However, the TEM sections from the edaravone group showed numerous glycogen granules and normal mitochondria. Furthermore, the glycogen granules in edaravone group were strong and had dark appearance.

Statistical comparison for the density of glycogen granules is shown in - Fig. 2. The mean density of glycogen granules in the edaravone group was significantly higher than that in the control group $\left(88.5 \pm 5.3\right.$ vs. $16.4 \pm 3.1$ particles $/ \mu^{2}$, $p<0.001)$. Edaravone reduced loss of glycogen because of reperfusion injury.

\section{Discussion}

Edaravone is free radical scavenger, now commercially available for acute cerebral infarction since 2001 in Japan. In addition to more suppressive effect in acute cerebral infraction, the experimental study shows that edaravone suppress postoperative spinal cord infarction ${ }^{7}$ and also protect cerebral damage during aortic arch surgery. ${ }^{8}$ Moreover, edaravone was reported to attenuate abdominal aortic aneurysms in apolipoprotein E-deficient mice. ${ }^{9}$ Using rat models, we also showed that edaravone might suppress reperfusion injury. ${ }^{4,5}$ And, our recent study of PAS staining indicated that edaravone maintained high glycogen storage in muscles. ${ }^{6}$ However, we did not evaluate the glycogen granule itself.

The mitochondria provide the source of energy for the cell. TEM can give us a lot of ultrastructural information about the

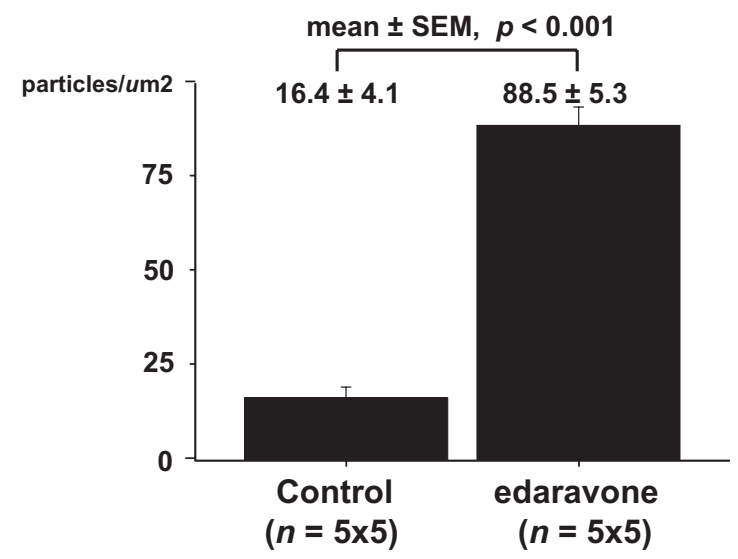

Fig. 2 The comparison of glycogen storage (density of glycogen granules), control group versus edaravone group. 
cell. Recently, using TEM at magnification of $\times 30,000$, we found edaravone reduced the mitochondrial swelling, which is the start of reperfusion injury. ${ }^{6}$ In this study, we focused on glycogen granules using TEM at higher magnification of $\times 50,000$. And we found that the muscles in edaravone group maintained significantly higher glycogen granules. Therefore, we have confirmed that edaravone can suppress reperfusion injury in rat models, by protecting the good energy consumption for the cell.

Regarding the timing of administration, edaravone may be given after declamping in the clinical settings. Now, we are investigating whether edaravone is as effective given after declamping.

\section{Note}

This study was presented at Vascular Annual Meeting, Chicago in June, 2011.

\section{Acknowledgments}

We thank Mitsubishi Tanabe Pharma Co., Japan for kindly donating edaravone. We also wish to thank Ritsuko Fujimoto, Hirotsugu Kubo (Research Facility for Common Use Joint-Use Research Facilities, Hyogo College of Medicine), and Tomokazu Kawakami (The Institute of Experimental Sciences, Hyogo College of Medicine) for their technical supports. This study is supported by Grant-in-Aid for Researchers, Hyogo College of Medicine 2010-2011, 2012.

\section{References}

1 Haimovici H. Arterial embolism with acute massive ischemic myopathy and myoglobinuria: evaluation of a hitherto unreported syndrome with report of two cases. Surgery 1960; 47:739-747

2 Haimovici H. Metabolic complications of acute arterial occlusions and skeletal muscle ischemia: Myonephropathic-metabolic syndrome. In: Haimovici H, ed. Haimovici's Vascular Surgery, 4th ed. Blackwell Science Inc.: Malden, Massachusetts; 1996: 509-530

3 Watanabe T, Morita I, Nishi H, Murota S. Preventive effect of MCI186 on 15-HPETE induced vascular endothelial cell injury in vitro. Prostaglandins Leukot Essent Fatty Acids 1988;33(1):81-87

4 Yamamura M, Miyamoto Y, Mitsuno M, et al. Suppression of rat lower extremity postoperative reperfusion injury with edaravone. Int J Angiol 2006;15(1):34-36

5 Yamamura M, Miyamoto Y, Mitsuno M, et al. Edaravone suppresses postoperative reperfusion injury in rat lower extremity: An immunohistological study. Int J Angiol 2007;16(1):17-19

6 Yamamura M, Miyamoto Y, Mitsuno M, et al. Edaravone reduces mitochondrial damage due to reperfusion injury following leg ischemia in rats. Int J Angiol 2010;19(4):e129-e131

7 Chiba K, Makuuchi H, Murakami H, et al. Effects of edaravone on prevention of paraplegia caused by ischemic spinal cord. Jpn J Cardiovasc Surg 2008;37(2):82-90 (Abst in English)

8 Kitanaka Y, Makuuchi H, Murakami H, et al. Effects of edaravone on cerebral protection during aortic arch surgery. Jpn J Cardiovasc Surg 2011;40(2):48-53 (Abst in English)

9 Uchida HA, Takatsuka T, Shikata K, et al. Edaravone attenuate angiotensin II-induced abdominal aortic aneurysms in apolipoprotein E-deficient mice. Arteriosclerosis Thrombosis and Vascular Biology 2011 Scientific Sessions. Program Abstr 2011:58 